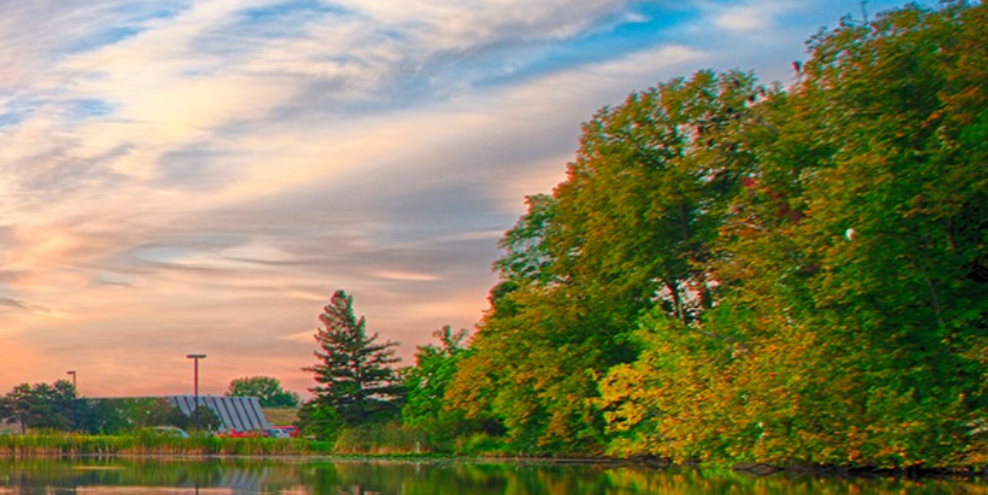

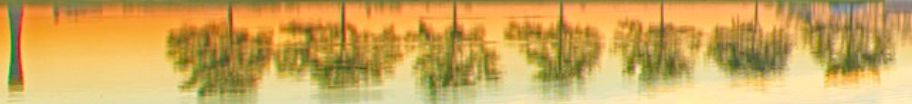

\title{
Static Analyzer Non-Comprehensive Overview
}

Dr Christopher Jones

HOW 2019

21 March 2019

This manuscript has been authored by Fermi Research Alliance, LLC under Contract No. DE-AC02-07CH11359 with the U.S. Department of Energy, Office of Science, Office of High Energy Physics 


\section{Purpose of Talk}

- Provide an overview of some of the code static analysis done by experiments

- Not a comprehensive list

- I only contacted people I knew

- Any mistakes in the information presented are mine

- I list all CMS ones though other experiments may have similar checkers

- Meant to start a discussion in the meeting 


\section{Compiler}

- Experiments known to use: all

- Warnings from the compiler are a form of static analysis

- Many experiments use multiple compilers or versions of a compiler

- clang and gcc seem to be the most popular 


\section{Coverity}

- Experiments known to use: ATLAS, CMS, LHCb

- Commercial package

- https://www.synopsys.com/software-integrity/security-testing/static-analysis-sast.html

- CERN has a license

- Provides a wide selection of sanity and correctness checking for $\mathrm{C}++$

- improper memory handle

- many kinds of resource leaks

- failing to release file handles

- threading problems

- deadlocks

- improper locking

- Has had problems keeping up with the $\mathrm{C}++$ standard

- All known experiments have temporarily stopped using it because of this 


\section{Codacy}

- Experiments Known to Use: ALICE

- Commercial Tool

- https://www.codacy.com

- https://github.com/marketplace/codacy

- Provides tools for automating code reviews

- Uses a plugin system to run different tools for multiple languages

- cppcheck

- flawfinder

- Pylint

- Easy integration with GitHub

- Nice reporting tools 


\section{cppcheck}

- Experiments known to use: ALICE, ATLAS

- Open Source

- http://cppcheck.sourceforge.net

- Reports bug in $\mathrm{C} / \mathrm{C}++$ with an emphasis on undefined behavior

- dead pointers

- integer overflows

- invalid use of STL 


\section{clang-tidy}

- Experiments Known to Use: ALICE, CMS

- Open source

- https://clang.|lvm.org/extra/clang-tidy/

- stand alone executable

- Can diagnose and in some cases fix typical programming errors

- add override keyword

- change comparison of std: : string to "' to call to empty()

- Very customizable via configuration

- Can be extended

- Examples from ALICE

- enforce member data naming convention

- catch cases where sizeof should be used 


\section{clang Static Analyzer}

- Experiment known to use: CMS

- Open source

- Plugins loaded by the clang compiler

- Uses exhaustive program-flow to try to find problems

- returning null reference

- dead assignment

- memory leaks

- CMS extensions

- using namespace in headers

- lots of thread safety checks

- global variables

- const member functions returning non-const pointers to member data

- Use thread-safety report in conjunction with a graph of what functions call other functions to find all Framework modules associated with 'global' variables 


\section{gcc plugin}

- Experiment known to use: ATLAS

- Open source

- plugins loaded by the gcc compiler

- ATLAS uses

- enforcing naming conventions

- flagging thread-unsafe constructs

- mark code as being required to be thread safe using $\mathrm{C}++$ annotations

- marked code can only call other marked code 


\section{Include What You Use}

- Experiment known to use: CMS

- Open source

- https://github.com/include-what-you-use/include-what-you-use

- based on clang

- Can identify and fix incorrect includes

- unneeded headers

- missing direct includes for cases where functions/classes are indirectly included 


\section{gcc libCheck}

- Experiment known to use: CMS

- Open source

- gcc using -as-needed flag

- makes linker say which linked libraries were unnecessary 


\section{CMS Homegrown}

- Package dependency checker

- packages are the smallest unit CMS uses to compile

- attempt to enforce allowed dependencies between groups of packages

- e.g. Reconstruction code should not dependent on simulation

- Checks for ROOT dictionaries

- find duplicate ROOT dictionaries across packages

- find dictionaries defined in a package not containing the C++ class

- catch class changes without corresponding ROOT version number change 